\title{
Mitigative Effects of Alpha-lipoic Acid on the Toxicity of Dimethoate in Male Rats
}

\author{
Hani M. Abdelsalam
}

\section{Hani M. Abdelsalam* \\ Department of Zoology, Faculty of Science, Zagazig University, Ash Shar- qia Governorate, EGYPT. \\ Correspondence}

\section{Hani M. Abdelsalam}

Department of Zoology, Faculty of Science, Zagazig University, Ash Sharqia Governorate, EGYPT.

Email: hmmsama@hotmail.com

\section{History}

- Submission Date: 04-06-2019

- Review completed: 09-07-2019;

- Accepted Date: 18-07-2019.

\section{DOI : 10.5530/ijcep.2019.6.2.15}

\section{Copyright}

(c) 2019 Phcog.Net. This is an openaccess article distributed under the terms of the Creative Commons Attribution 4.0 International license.

\begin{abstract}
Background and Aim: Organophosphates are currently widely used by humans, but these compounds have tremendous negative effects on human health. Therefore, this study aims to use alpha-lipoic acid (ALA) to alleviate the negative effects of dimethoate (DM). Methods: This study was designed as follows: Thirty adult male Wistar albino rats were utilized and further subdivided into control, DM and DM+ALA groups. Liver and renal cortex sections from all groups were processed for histopathological examination and biochemical estimation of liver function tests, serum urea, creatinine, BUN, testosterone and lipid profile was performed. Results: This study clarified the ameliorative effects of ALA on the negative effects of DM, where ALA induced a significant recovery of hepatic (ALT, AST, ALP and total protein) and renal functions by normalizing them in the DM + ALA group and promoted some improvement of lipid profile and testosterone levels. Additionally, ALA restored normal hepatic and renal histomorphology. Conclusion: ALA therapy can be concluded to ameliorate the negative effects of DM that affect vital organs such as the liver and kidney. Additionally, ALA can reduce the occurrence of atherogenesis by decreasing the levels of unhealthy cholesterol in the blood. ALA also boosts testosterone levels, thus augmenting male sexual characteristics.

Key words: Organophosphates, Dimethoate, Alpha-lipoic acid, Dihydrolipoic acid, Antioxidant.
\end{abstract}

\section{INTRODUCTION}

Organophosphates (OPs), such as dimethoate (DM), have been a serious danger since World War II and have major involvement in a predicted thirty thousand instances of severe pesticide poisoning reported worldwide. ${ }^{[1]}$ One main effect of this group is the inhibitory effect on acetylcholinesterase (AChE), ${ }^{[2]}$ and the possible implication that it prompts alterations in characteristics of oxidative stress. ${ }^{[3]}$ The same appears to be the case for dimethoate, since these compounds are known to increase the production of reactive oxygen species (ROS) and reduce the antioxidant barrier of the organism to induce oxidative stress. ${ }^{[1]}$

Repetitive or continued exposure to OPs may result in many effects, such as acute exposure, including delayed symptoms. Workers repeatedly exposed to DM reported impaired memory, disorientation, depression, irritability, confusion, headache, speech difficulties, delayed reaction times, nightmares, sleepwalking and drowsiness or insomnia. Additionally, influenza-like conditions with headache, nausea, weakness, loss of appetite and malaise have also been reported. ${ }^{[4]}$ Dimethoate disturbs the functions of multiple organs, including the liver. ${ }^{[5]}$ The liver is the primary organ involved in metabolism and is a target organ of OPs and drugs. Clinical biochemis-

try and histopathological evaluations of the liver are the methods used to discover the exposure effects of OPs. ${ }^{[6]}$

Alpha-lipoic acid (ALA) is a naturally occurring dithiol compound that serves as a vital cofactor for mitochondrial bioenergetic enzymes ${ }^{[7]}$ Interestingly, ALA supplementation reduced renal injury in rats with obstructive nephropathy, further indicating that oxidative stress inhibition is likely to be involved in the beneficial effects of this compound. ${ }^{[8]}$ The therapeutic potential of ALA has been proved in a variety of disorders linked to oxidative stress and inflammation in diverse organ systems, including the kidney. ${ }^{[9]}$ ALA and its reduced dithiol form, dihydrolipoic acid, are potent antioxidants that can scavenge hydroxyl radicals, singlet oxygen, hydrogen peroxide, hypochlorous acid, peroxynitrite and nitric oxide and these categories are much broader than those of vit E. The ALA/DHLA redox couple can regenerate exogenous and endogenous antioxidants such as vit $\mathrm{C}$ and $\mathrm{E}$ and Glutathione (GSH). Additionally, the couple exerts additional antioxidant actions through the chelation of copper, iron and other transitional metals. ${ }^{[10]}$ ALA alleviated histopathologic and morphologic lesions in extracted rat kidneys. Addition-

Cite this article: Abdelsalam HM. Mitigative Effects of Alpha-lipoic Acid on the Toxicity of Dimethoate in Male Rats. Int J Clin Exp Physiol. 2019;6(2):55-60. 
ally, a positive effect of ALA on albuminuria was detected. However, no beneficial effect of ALA was found on antioxidant parameters at the tissue level. ${ }^{[11]}$

\section{MATERIALS AND METHODS}

\section{Experimental Animals}

Thirty healthy adult male Wistar albino rats (11-13 weeks) weighing 160-180 g were obtained and maintained at the Breeding Animal House of the Faculty of Medicine, Zagazig University, Egypt. Animals were housed in plastic cages at a controlled temperature $\left(25 \pm 1^{\circ} \mathrm{C}\right)$ and humidity level $(56 \pm 5 \%)$ in an artificially illuminated room, completely free from chemical contamination. They were fed with standard laboratory food and had free access to food and water. All rats received humane care in compliance with the Ethical Committee of Zagazig University and in accordance with the National Institutes of Health (NIH) Guidelines for the Care and Use of Laboratory Animals.

\section{Experimental Design}

The animals were assigned to 3 groups as follows:

Group I (control group): 10 rats that received no treatment for 2 months. Group II (DM group): 10 rats that received dimethoate $(20 \mathrm{mg} / \mathrm{kg}$ body weight) dissolved in $1 \mathrm{ml}$ corn oil [1/20 of the $\operatorname{LD} 50(380 \mathrm{mg} / \mathrm{kg})]^{[12]}$ once daily for 14 weeks via oral intubation.

Group III (DM+ALA group): 10 rats that received dimethoate $(20 \mathrm{mg} /$ $\mathrm{kg}$ body weight) dissolved in $1 \mathrm{ml}$ corn oil ( $380 \mathrm{mg} / \mathrm{kg}$ ) once daily for 14 weeks via oral intubation ${ }^{[12]}$ and then received ALA $(50 \mathrm{mg} / \mathrm{kg}){ }^{[13]}$

At the end of the experiment, blood samples were collected, ${ }^{[14]}$ and then rats in all groups were sacrificed via intraperitoneal injection of $25 \mathrm{mg} / \mathrm{kg}$ sodium thiopental. ${ }^{[15]}$ Liver and kidney sections were collected and fixed. Samples were processed for light microscope examination.

\section{Experimental Procedures}

Dimethoate (purity 98\%) and N-acetylcysteine were purchased from Sigma Chemicals, St. Louis, MO, USA. Alpha-lipoic acid capsules (thiotex forte $600 \mathrm{mg} /$ capsule) were obtained from Marcyrl Pharmaceutical Industries, El-obour city, Egypt.

\section{Collection of blood samples}

Blood samples were collected from the retro-orbital venous plexus under mild anaesthesia using a fine heparinized capillary tube introduced into the medial epicanthus of the rat's eye. Blood samples were collected in a clean graduated centrifuge tube, allowed to clot at room temperature for $10 \mathrm{~min}$ and then centrifuged using a Remi cool centrifuge at $3000 \mathrm{rpm}$ for $20 \mathrm{~min}$. The supernatant was collected in a dry tube for assessment of biochemical parameters. All biochemical procedures were conducted in the Central Research Laboratory, Faculty of Medicine, Zagazig University.

\section{Biochemical estimation of serum urea, creatinine and BUN}

Serum urea was assessed by a quantitative colorimetric method using QuantiChrom ${ }^{\mathrm{TM}}$ assay kits (BioAssay Systems, USA). Blood urea nitrogen (BUN) was measured using a commercial kit (BUN II reagent kit; Wako Pure Chemical Industries). ${ }^{[16]}$

\section{Biochemical estimation of serum lipid profile}

Triglycerides, total cholesterol, HDL-cholesterol and LDL-cholesterol concentrations were evaluated enzymatically using assay kits (Sigma Chemical Co, St Louis, MO, USA). Very low-density lipoprotein (VLDL)-cholesterol was calculated as triglycerides and LDL-cholester- ol was calculated by the equation LDL-cholesterol=Total serum cholesterol $-(\mathrm{HDL}+\mathrm{VlDl}) \cdot{ }^{[17]}$

\section{Biochemical estimation of serum ALT, AST, ALP and TP.}

The activities of serum alanine aminotransferase (ALT), Alkaline Phosphatase (ALP) and Aspartate Aminotransferase (AST) were measured Spectrophotometrically using an automated analyser method (Opera Technician Bayer Autoanalyzer). The serum Total Protein (TP) level was determined by the colorimetric point method.

\section{Histopathological Study}

All steps were conducted in the Histology and Cell Biology Department, Faculty of Medicine, Zagazig University. Specimens from the liver and renal cortex of each animal were fixed in $10 \%$ neutral formol saline, dehydrated, embedded in paraffin wax and processed into $5 \mu \mathrm{m}$ thick sections. The sections were stained with haematoxylin and eosin. Stained slides were analysed by light microscopy in Image Analysis Unit. ${ }^{[18]}$

\section{Statistical Analysis}

Data were documented and entered using the statistical package SPSS version 13. Data were described using the mean and standard error for quantitative variables. Comparisons between groups were performed using one-way analysis of variance with multiple comparisons post hoc testing. ${ }^{[19]}$ The results were considered statistically significant at $P<$ 0.05 .

\section{RESULTS}

\section{Biochemical estimation of serum liver functions}

The DM-treated group revealed a highly significant increase in the mean ALT, AST and ALP levels compared to the control group $(P<0.01)$, although it showed a significant decrease in the mean TP level. The DM+ALA group exhibited a significant decrease in the mean ALT, AST and ALP $(P<0.01)$ levels compared to the DM-treated group, while it showed a significant increase in the mean TP level $(P<0.05)$. Statistical results of the various studied groups are presented in Table 1.

\section{Biochemical estimation of serum urea, creatinine, BUN and testosterone}

Table 2 shows that the DM-treated group demonstrated a highly significant increase in the mean urea level compared to the control group $(P<0.01)$, while it showed a significant increase $(P<0.05)$ in mean creatinine and a highly significant decrease in testosterone levels. The $\mathrm{DM}+\mathrm{ALA}$ group showed a significant decrease in the mean urea, creatinine and BUN concentrations $(P<0.01)$ compared to the DM-treated group, while it showed a significant increase in the mean testosterone level $(P<0.01)$.

\section{Biochemical estimation of serum lipid profile}

The DM-treated group exhibited a statistically significant increase in the mean TG, LDL-c, VLDL-c and atherogenic index compared to the control group $(P<0.05)$, while it showed a non-significant decrease in the mean TC and HDL-c levels. The DM+ALA group revealed a nonsignificant lower mean TG, LDL-c and atherogenic index compared to the DM-treated group $(P>0.05)$. Moreover, a statistically significant increase in TC level $(P<0.05)$ and a non-significant increase in HDL-c and VLDL-c levels $(P>0.05)$ were observed. Statistical results of the various studied groups are presented in Table (3). 
Table 1: Serum TP, ALT, AST and ALP in the various studied groups.

$\begin{array}{ccccc} & & \text { Control } & \begin{array}{c}\text { Dimethoate } \\ (\text { DM })\end{array} & \text { DM+ALA } \\ & & & 5.32 \pm 0.37 & 6.51 \pm 0.28 \\ \text { T. protein } & \text { Mean } \pm \text { SE } & 6.76 \pm 0.19 & -21.30^{\mathrm{a}} & +22.36^{\mathrm{c}} \\ (\mathrm{TP})(\mathrm{g} / \mathrm{dl}) & \% \text { change } & & <0.01^{* * a} & <0.05^{* c} \\ & \text { Significance } & & 23.72 \pm 1.52 & 16.53 \pm 0.74 \\ & \text { Mean } \pm \text { SE } & 15.39 \pm 1.02 & +54.13^{\mathrm{a}} & -30.31^{\mathrm{c}} \\ \text { ALT } & \text { \% change } & & <0.01^{* * a} & <0.001^{* * c} \\ (\mathrm{IU} / \mathrm{L}) & \text { Significance } & & 21.77 \pm 1.39 & 15.65 \pm .26 \\ & \text { Mean } \pm \text { SE } & 16.56 \pm 0.94 & +31.46^{\mathrm{a}} & -28.11^{\mathrm{c}} \\ \text { AST } & \% \text { change } & & <0.01^{* * a} & <0.01^{* * c} \\ (\mathrm{IU} / \mathrm{L}) & \text { Significance } & & 120.97 \pm 6.64 & 76.73 \pm 4.56 \\ & \text { Mean } \pm \text { SE } & 70.48 \pm 1.84 & +71.64^{\mathrm{a}} & -36.57^{\mathrm{c}} \\ \text { ALP } & \text { \% change } & & <0.01^{* * a} & <0.01^{* * c}\end{array}$

${ }^{*} P<0.05$ : Significant, ${ }^{*} P<0.01$ : Highly significant and $P>.05$ : Non-Significant ${ }^{a}$ is comparison of control vs DM, $c$ is comparison of DM vs DM+ALA

Table 3: Serum Lipid profile in various studied groups.

\begin{tabular}{|c|c|c|c|c|}
\hline & & Control & $\begin{array}{l}\text { Dimethoate } \\
\text { (DM) }\end{array}$ & $\mathrm{DM}+\mathrm{ALA}$ \\
\hline \multirow{3}{*}{$\mathrm{TC}(\mathrm{mg} / \mathrm{dl})$} & Mean \pm SE & $151.01 \pm 6.30$ & $150.25 \pm 9.14$ & $178.37 \pm 4.78$ \\
\hline & $\%$ change & & $-0.50^{\mathrm{a}}$ & $+18.71^{\mathrm{c}}$ \\
\hline & Significance & & $>.05^{\mathrm{a}}$ & $<0.05^{\star c}$ \\
\hline \multirow{3}{*}{ TG (mg/dl) } & Mean \pm SE & $183.25 \pm 2.92$ & $211.37 \pm 12.06$ & $192.62 \pm 10.85$ \\
\hline & $\%$ change & & $+15.34^{\mathrm{a}}$ & $-8.87^{c}$ \\
\hline & Significance & & $<0.05^{\star a}$ & $>.05^{c}$ \\
\hline \multirow{3}{*}{$\begin{array}{l}\text { HDL-C } \\
(\mathrm{mg} / \mathrm{dl})\end{array}$} & Mean \pm SE & $54.75 \pm 2.36$ & $44.50 \pm 12.05$ & $50.62 \pm 2.37$ \\
\hline & $\%$ change & & $-18.72^{\mathrm{a}}$ & $+13.75^{\mathrm{c}}$ \\
\hline & Significance & & $>.05^{\mathrm{a}}$ & $>.05^{c}$ \\
\hline \multirow{3}{*}{$\begin{array}{l}\text { LDL-C (g/ } \\
\text { dl) }\end{array}$} & Mean \pm SE & $161.76 \pm 10.15$ & $200.23 \pm 9.04$ & $182.25 \pm 2.05$ \\
\hline & $\%$ change & & $+23.78^{\mathrm{a}}$ & $-8.97^{c}$ \\
\hline & Significance & & $<0.05^{\star \mathrm{a}}$ & $>.05^{c}$ \\
\hline \multirow{3}{*}{$\begin{array}{l}\text { VLDL-C } \\
(\mathrm{mg} / \mathrm{dl})\end{array}$} & Mean \pm SE & $34.77 \pm 0.70$ & $42.35 \pm 2.66$ & $43.51 \pm 2.30$ \\
\hline & $\%$ change & & $+21.80^{\mathrm{a}}$ & $+2.73^{\mathrm{c}}$ \\
\hline & Significance & & $<0.05^{* a}$ & $>.05^{\mathrm{c}}$ \\
\hline \multirow{3}{*}{$\begin{array}{l}\text { Atherogenic } \\
\text { index }\end{array}$} & Mean \pm SE & $0.183 \pm 0.02$ & $0.253 \pm 0.02$ & $0.224 \pm 0.02$ \\
\hline & $\%$ change & & $+38.25^{\mathrm{a}}$ & $-11.46^{c}$ \\
\hline & Significance & & $<0.05^{\text {*a }}$ & $>.05^{c}$ \\
\hline
\end{tabular}

${ }^{\star} P<0.05$ : Significant, ${ }^{*} P<0.01$ : Highly significant and $P>0.05$ : Non-Significant ${ }^{a}$ is comparison of control vs DM, $c$ is comparison of DM vs DM+ALA

\section{DISCUSSION}

In a previous study, after dimethoate exposure, serum levels of lipid peroxidation, catalase (CAT), glutathione reductase and superoxide dismutase activities (SOD) were increased. ${ }^{[20]} \mathrm{DM}$ affects the functions of multiple organs, including the liver. It was reported to change the level
Table 2: Serum urea, creatinine, BUN and testosterone in various studied groups.

\begin{tabular}{|c|c|c|c|c|}
\hline & & Control & $\begin{array}{c}\text { Dimethoate } \\
\text { (DM) }\end{array}$ & $\mathrm{DM}+\mathrm{ALA}$ \\
\hline \multirow{3}{*}{ Urea $(\mathrm{mg} / \mathrm{dl})$} & Mean \pm SE & $56.67 \pm 2.55$ & $83.89 \pm 1.85$ & $48.22 \pm 3.43$ \\
\hline & $\%$ change & & $+48.03^{\mathrm{a}}$ & $-42.51^{c}$ \\
\hline & Significance & & $<0.01^{* * a}$ & $<0.01^{* * c}$ \\
\hline \multirow{3}{*}{$\begin{array}{l}\text { Creatinine } \\
(\mathrm{mg} / \mathrm{dl})\end{array}$} & Mean \pm SE & $0.63 \pm 0.009$ & $0.79 \pm 0.04$ & $0.63 \pm 0.60$ \\
\hline & $\%$ change & & $+25.39^{\mathrm{a}}$ & $-20.25^{c}$ \\
\hline & Significance & & $<0.05^{\star a}$ & $<0.01^{* * c}$ \\
\hline \multirow{3}{*}{$\mathrm{BUN}(\mathrm{mg} / \mathrm{dl})$} & Mean \pm SE & $25.94 \pm 1.14$ & $26.88 \pm 1.48$ & $19.97 \pm 1.05$ \\
\hline & $\%$ change & $+3.62^{\mathrm{a}}$ & $-25.70^{c}$ & \\
\hline & Significance & $>.05^{\mathrm{a}}$ & $<0.01^{\star \star c}$ & \\
\hline \multirow{3}{*}{$\begin{array}{l}\text { Testosterone } \\
\text { (nM/L) }\end{array}$} & Mean \pm SE & $4.42 \pm 0.12$ & $3.48 \pm 0.09$ & $4.35 \pm 0.08$ \\
\hline & $\%$ change & & $-21.26^{\mathrm{a}}$ & $25^{c}$ \\
\hline & Significance & & $<0.01^{* * a}$ & $<0.01^{* * c}$ \\
\hline
\end{tabular}

${ }^{\star} P<0.05$ : Significant, ${ }^{*} P<0.01$ : Highly significant and $P>0.05$ : Non-Significant ${ }^{a}$ is comparison of control vs DM, $c$ is comparison of DM vs DM+ALA

of liver-related marker parameters in rats and mice. ${ }^{[5]}$ Numerous studies show that dimethoate intoxication can cause oxidative stress by the generation of free radicals and induce hepatic lipid peroxidation in mice ${ }^{[21]}$ and rats. ${ }^{[12]}$ Our findings corroborate with these reports as we found significant elevation in the levels of liver enzymes (ALT, AST and ALP) on administration of DM for $(20 \mathrm{mg} / \mathrm{kg}$ b.wt.) for 14 weeks.

These results were consistent with another report, ${ }^{[22]}$ where a marked increase in ALT, AST and ALP in the serum of the dimethoate-treated group was recorded. In addition, changes in protein metabolic profiles were dose- and time-dependent. According to Attia et al. ${ }^{[20]}$ Serum TP, albumin and globulin were reduced and bilirubin levels were elevated in dimethoate-treated rats. The decline in serum protein could be attributed to alterations in protein and free amino acid metabolism and their synthesis in the liver. ${ }^{[23]}$ Additionally, the diminishing protein level may be due to loss of protein either by a decrease in protein synthesis or increase in proteolytic activity or degradation. ${ }^{[24]}$ In light of current knowledge, the present results showed a significant reduction in total protein levels after treatment of the rats with DM. This finding was similar to reports by Attia et al. ${ }^{[20]}$ Histopathological results revealed a substantial DM-induced impairment of the architecture of the hepatocytes and liver constituents, where degenerated hepatocytes, pyknotic nuclei, focal fibrosis, dilated biliary ducts and inflammatory infiltrations appeared in the DM group, as illustrated in Figure 1. These observations are in agreement with Alarami et al. ${ }^{[25]}$ who elucidated that mice exposed to DM showed progressive changes in the liver, including congested blood vessels, infiltration, vasodilatation and hydropic alterations. It was previously found that DM can produce changes in the liver, such as lymphocytic infiltration, congestion, nuclear death, enlargement of hepatic sinusoids, hepatocellular damage, cytoplasmic vacuolation and degeneration in nuclei. These alterations are caused by changes in cellular integrity and membrane permeability due to exposure to toxic chemicals. ${ }^{[26]}$ Additionally, DM, which is a lipophilic substance, can interact with cellular membranes, ${ }^{[27]}$ and membrane injury or necrosis discharges such enzymes into the circulation. ${ }^{[26]}$ ALA administration restored the normal architecture of the hepatic strands, hepatocytes and nuclei. The portal 
Histopathological results
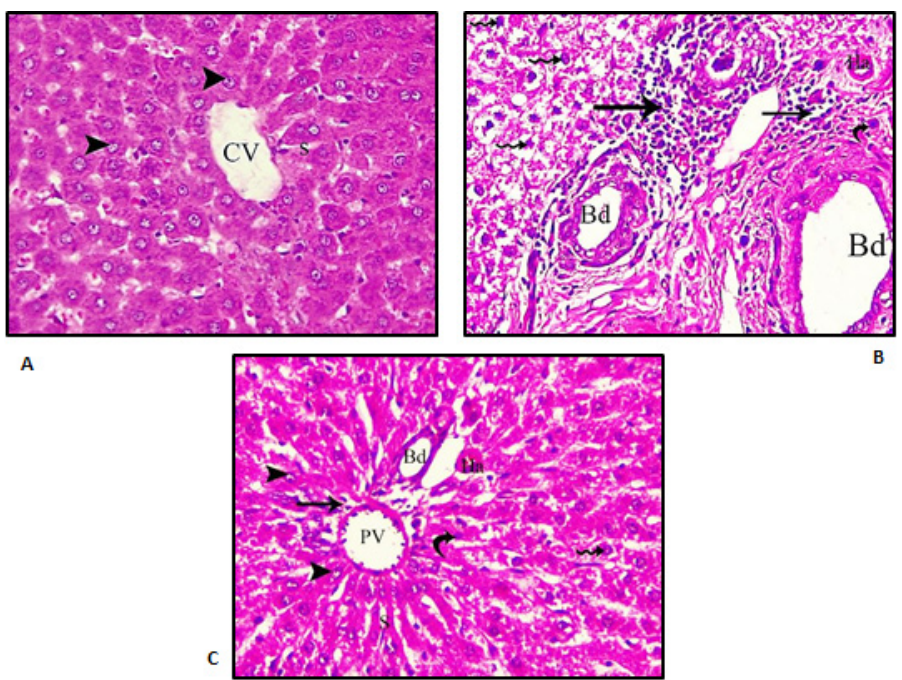

Figure 1: Photomicrographs of sections of the liver from adult male rats in different groups. (A): Control group showing normal hepatic laminae, hepatocytes and intact nuclei (arrowheads). The Central Vein (CV) lies at the centre of the lobule surrounded by the hepatocyte strands (with strongly eosinophilic granulated cytoplasm), which are separated by blood sinusoids (S). (B): DM group showing focal necrosis with fibrosis and inflammatory infiltration (arrow). Additionally, congestion of hepatic sinusoids and a dilated Bile Duct (Bd) were observed. Hepatocytes have lost their polygonal architecture and have a vacuolated cytoplasm with deeply stained pyknotic nuclei (zigzag arrow), while the hepatic artery $(\mathrm{Ha})$ is congested and apoptotic bodies (curved arrow) also appeared. (C): DM+ALA group showing a restoration of normal architecture of the hepatic strands, hepatocytes and normal nuclei (arrowheads). The portal vein (PV) and Bile Duct (Bd) appeared normally shaped, while apoptotic bodies (curved arrow) were considerably decreased.

vein and bile duct appeared normal, while apoptotic bodies were considerably decreased.

Several animal studies have stated that ALA has blood lipid-moderating properties beyond its antioxidant and anti-inflammatory features. ${ }^{[28]}$ ALA has been confirmed to have various useful impacts, preventing and treating many diseases through its antioxidant and anti-inflammatory activities. ${ }^{[29]}$ ALA is a disulfide compound that serves as a coenzyme in pyruvate dehydrogenase and alpha-ketoglutarate dehydrogenase mitochondrial reactions, causing the production of cellular energy (ATP). ${ }^{[30]}$ The protective properties of ALA against hepatotoxicity and oxidative stress has been well recognized in a series of scientific reports. ${ }^{[31]}$ Our results showed that administration of ALA to DM-treated rats caused significant recovery of liver-related biochemical markers, in which ALT, AST and ALP were recovered to approximately normal levels, as shown in Table (1). These results were in line with Valdecantos et al. ${ }^{[32]}$ Who proved that adding ALA to the diet of rats receiving a high-fat diet resulted in a noticeable reduction in ALT and AST. These findings are also supported by Evans et al. ${ }^{[33]}$ who reported that ALA and its reduced form, dihydrolipoic acid, decrease oxidative stress by scavenging a number of free radicals in both membrane and aqueous areas by stopping membrane lipid peroxidation and protein damage through the redox regeneration of other antioxidants, such as vitamins $\mathrm{C}$ and $\mathrm{E}$ and by enriching intracellular glutathione. ${ }^{[33]}$ In addition to its role as an antioxidant, ALA can change the activity of enzymes at various levels in metabolic pathways. Additionally, ALA can alter mammalian pyruvate dehydrogenase complex (PDC) based on its stereoselectivity. ${ }^{[34]}$ Therefore, in our study, ALA might have restored hepatic enzymatic activity by acting through any of these pathways.

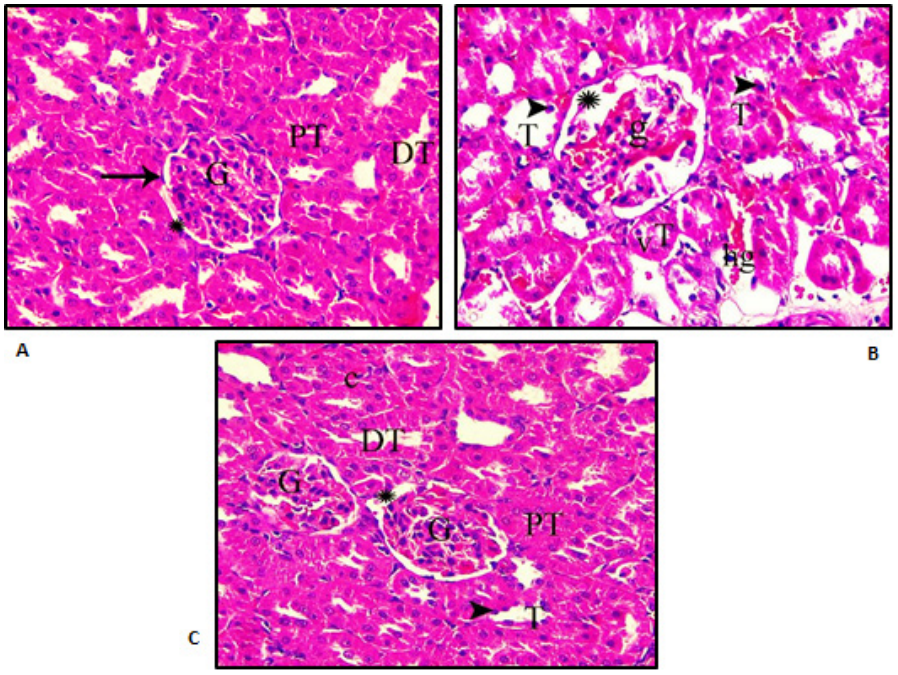

Figure 2: Photomicrographs of sections of the renal cortex from adult male rats in different groups. (A): Control group showing normal glomeruli $(G)$, Bowman's capsules lined by intact simple squamous cells (arrow), narrow Bowman's space (star), proximal convoluted tubules (PCT) with cuboidal epithelium and narrow lumen (PT) and Distal Convoluted Tubules (DCT) with wide lumen (DT). (B): DM group showing a shrunken and segmented glomerulus (g), marked haemorrhaging (hg) at distal convoluted tubules and wide Bowman's spaces (star). Renal tubules (T) reveal exfoliated cells with dark-stained apoptotic nuclei (arrowhead) and vacuolation (V) of renal tubule cells. (C): $D M+A L A$ group showing nearly normal glomeruli $(G)$ but with wide Bowman's spaces (star). Some tubules (T) appear with darkly stained nuclei (arrowhead) and capillaries between tubules (c). Normal proximal convoluted tubules (PT) and distal convoluted tubules (DT) are seen.

Organophosphorus prompts $\mathrm{H} 2 \mathrm{O} 2$ production and lipid peroxidation in kidney cells. ${ }^{[35]}$ Others have provided additional evidence for the existence of organophosphorus-induced oxidative tissue injury evidenced by DNA-strand breaks, augmented activities of antioxidant enzymes ${ }^{[36]}$ and downregulation of glutathione peroxidase activity and glutathione. ${ }^{[37]}$ Lipid peroxidation, ATP reduction, DNA destruction, protein oxidation and intracellular calcium increases due to membrane permeability injuries may be the pathway for renal cell damage by organophosphorus. ${ }^{[38-41]}$ Creatinine is an amino acid formed as a waste product of creatine, which acts as a vital energy reservoir in muscle metabolism. Urea and creatinine levels in blood increase during kidney impairment, preventing the kidneys from filtering urea and creatinine out of the blood. Lone et al. ${ }^{[39]}$ Reported a significant increase in urea and creatinine levels in serum after DM exposure for 15 and 30 days. These investigations suggested that DM prompted hepatic injury and specified that removal of waste products and toxins from the blood by the kidneys may suffer. ${ }^{[40]}$ Interestingly, our results showed that the levels of urea, creatinine and BUN were significantly increased in the DM group due to the induction of oxidative effects by DM, as shown in Table (2).

In the present study, ALA administration caused a substantial decrease in the mean levels of urea, creatinine and BUN, while it induced a significant increase in the mean testosterone level, as shown in Table (2). Previously, an animal model has shown that ALA protects against ischaemic acute renal failure, as exemplified by the attenuation in the level of BUN, creatinine, urinary osmolality, creatinine clearance (SCr) and fractional excretion of $\mathrm{Na}+$, as well as attenuation of tubular necrosis, proteinaceous casts and medullary congestion in renal tissue. The protective effects may be related in part to the decreased level of endothelin-1 (ET- 
1) in the kidney ${ }^{[42]}$ In another study, ALA increased reduced GSH, total antioxidant level and also $\mathrm{Na}+\mathrm{K}+$-ATPase activity. ${ }^{[43]}$ Therefore, the antioxidant restoring activity might be the mechanism for improvedkidney function in our study.

In our study Histopathological results of the kidney indicated that DM administration to rats caused destructive alterations in the architecture of the kidney, where DM caused shrunken and segmented glomeruli, marked haemorrhaging at the distal convoluted tubules and wide Bowman's spaces. Additionally, renal tubules revealed exfoliated cells with dark-stained apoptotic nuclei and vacuolation of renal tubule cells, as observed in Figure 2. These alterations are in line with the findings by Alarami et al. ${ }^{[25]}$ Who observed that DM caused histological alterations in the kidney, including glomerular degeneration, tubular degeneration, haemorrhaging, infiltration, hydropic changes, tubular casts, tubular widened lumen and glomerular shrinkage. In the ALA group, glomeruli were restored to nearly normal morphology but with wide Bowman's spaces. Additionally, normal proximal convoluted tubules and distal convoluted tubules were seen. A previous report by Şehirli et al. ${ }^{[43]}$ Showed that ALA regenerated and reduced tubular dilation and regenerated tubular epithelium, confirms our present findings. a-LA was reported to prevented eNOS and neuronal NOS production but diminished inducible NOS, while ALA decreased the expression levels of ET-1. ${ }^{[31]}$ Both in vitro and in vivo studies revealed that ALA reduced serum levels of BUN and SCr, MDA levels and ROS levels and ameliorated the severity of kidney injuries (tubular cell necrosis, cytoplasmic vacuolation, haemorrhage and tubular dilatation). ${ }^{[4]}$ Therefore, the same mechanisms might be the responsible for improved kidney function and morphology in our study.

The current research illustrated that the DM group exhibited a significant increase in TG, LDL-c, VLDL-c and atherogenic index compared to the control group, while HDL-c was decreased as shown in Table (3). This finding is in contrast to report by El-Saad et al. ${ }^{[45]}$ Who stated that DM caused an increase in serum total cholesterol levels. Increased serum cholesterol is due to the effects of pesticides on the permeability of liver cell membranes. ${ }^{[46]}$ The elevation in the level of serum total cholesterol may be or-due to obstruction of the liver bile ducts, reducing or preventing cholesterol secretion into the duodenum. ${ }^{[47]}$ An increase in the serum cholesterol level may be a sign of liver damage. ${ }^{[48]}$ El-Saad et al. ${ }^{[45]}$ also demonstrated that DM caused reductions in triglyceride and VLDL-cholesterol levels. Different pesticides decrease VLDL-cholesterol and triglyceride levels. ${ }^{[49]}$ Our finding is opposite to these reports.

Apart from the oxidative stress generated by DM, a significant reduction in the level of plasma HDL was observed, along with an increase in the levels of triglyceride, LDL and total cholesterol in the DM group. Free radicals can enhance the oxidation of LDL and oxidized LDL affects many biological processes involved in atherogenesis. In the ALA group, TG and LDL-c levels and the atherogenic index declined and returned to normal levels in the control group, but an increase in HDL-c and VLDLc occurred. This finding is in line with Morakinyo et al. ${ }^{[50]}$ Who clarified that ALA caused a significant increase in HDL levels with a concurrent significant reduction in TC, TG, LDL and VLDL (bad cholesterol) concentrations. Moreover Zhang et al.$^{[48]}$ Reported diminished serum TC, TG and LDL-C levels in obese subjects following ALA supplementation. The probable mechanism for these alterations is increasing insulin sensitivity and controlling the activities of the enzymes involved in lipolysis and triglyceride synthesis. ${ }^{[4]}$ Furthermore, ALA appears to increase the activities of lipoprotein lipase as well as lecithin cholesterol acyltransferase (LCAT). Overall ALA supplementation ameliorated all the biochemical and histological alterations caused by dimethoate administration in our study.

\section{CONCLUSION}

ALA therapy can ameliorate the negative effects of DM that affect vital organs, such as the liver and kidney. Additionally, ALA can reduce the occurrence of atherogenesis by reducing the levels of unhealthy cholesterol in the blood. Finally, ALA boosts levels of testosterone, augmenting male sexual characteristics.

\section{ABBREVIATIONS}

DM: Dimethoate; ALA: Alpha-lipoic acid; BUN: Blood urea nitrogen; TC: Total cholesterol; LDL: Low-density lipoprotein; HDL: High-density lipoprotein; TG: Triglycerides; OPs: Organophosphates; DHLA: Dihydrolipoic acid.

\section{Ethical approval and consent to participate}

All applicable international, national and/or institutional guidelines for the care and use of animals were followed and approved by Prof Dr. Abdallah Elhoot (Head of Zoology Department- Zagazig University)

\section{REFERENCES}

1. Lukaszewicz-Hussain A. Role of oxidative stress in organophosphate insecticide toxicity-Short review. Pesticide Biochemistry and Physiology. 2010;98(2):14550 .

2. Nazam $\mathrm{N}$, et al. Combined in silico and in vivo studies shed insights into the acute acetylcholinesterase response in rat and human brain. Biotechnology and Applied Biochemistry. 2015;62(3):407-15.

3. Shadnia $S$, et al. Evaluation of oxidative stress and genotoxicity in organophosphorus insecticide formulators. Human and Experimental Toxicology. 2005;24(9):439-45.

4. Ogutcu A, et al. The effects of organophosphate insecticide diazinon on malondialdehyde levels and myocardial cells in rat heart tissue and protective role of vitamin E. Pesticide Biochemistry and Physiology. 2006;86(2):93-8.

5. Khan AA, Shah MA, Rahman SU. Occupational exposure to pesticides and its effects on health status of workers in Swat, Khyber Pakhtunkhwa, Pakistan. Journal of Biology and Life Science. 2013;4(2):43-55

6. Crissman JW, et al. Best practices guideline: Toxicologic histopathology. Toxicologic Pathology. 2004;32(1):126-31.

7. Gorąca A, et al. Lipoic acid-biological activity and therapeutic potential. Pharmacological Reports. 2011;63(4):849-58.

8. Wongmekiat $\mathrm{O}$, Leelarungrayub $\mathrm{D}$, Thamprasert K. Alpha-lipoic acid attenuates renal injury in rats with obstructive nephropathy. Bio Med Research International. 2013;2013.

9. Kang KP, et al. Alpha-lipoic acid attenuates cisplatin-induced acute kidney injury in mice by suppressing renal inflammation. Nephrology Dialysis Transplantation. 2009;24(10):3012-20.

10. Bilska A, Wlodek L. Lipoic acid-the drug of the future. Pharmacol Rep 2005;57(5):570-7.

11. Ayhan M, et al. Preventive effects of alpha-lipoic acid on diabetic nephropathy in a rat model. Acta Medica Mediterranea. 2014;30(6):1221-5.

12. Kamath V, Joshi AKR, Rajini P. Dimethoate induced biochemical perturbations in rat pancreas and its attenuation by cashew nut skin extract. Pesticide Biochemistry and Physiology. 2008;90(1):58-65.

13. Emam AM, et al. Protective effects of alpha-lipoic acid and coenzyme Q10 on lipopolysaccharide-induced liver injury in rats. Pharm Lett. 2016;8(19):176-82.

14. Wang SJ, et al. Mechanism of treatment of kidney deficiency and osteoporosis is similar by Traditional Chinese Medicine. Current Pharmaceutical Design. 2016;22(3):312-20

15. Kara A, et al. Ultra-structural changes and apoptotic activity in cerebellum of post-menopausal-diabetic rats: A histochemical and ultra-structural study. Gynecological Endocrinology. 2014;30(3):226-31.

16. Roubalová $L$, et al. Flavonolignan 2, 3-dehydrosilydianin activates Nrf2 and upregulates NAD (P) H: Quinone oxidoreductase 1 in Hepa1c1c7 cells. Fitoterapia. 2017;119:115-20.

17. Chattopadhyay R, Bandyopadhyay M. Effect of Azadirachta indica leaf extract on serum lipid profile changes in normal and streptozotocin induced diabetic rats. African Journal of Biomedical Research. 2005;8(2):101-4

18. Bancroft JD, Layton C. The hematoxylins and eosin: Bancroft's Theory and Practice of Histological Techniques. Elsevier. 2013;173-86.

19. Armitage PG, Berry JNS, Matthews MA. Statistical methods in medical research. John Wiley and Sons. 2008;1-7.

20. Attia AM, Nasr H. Dimethoate-induced changes in biochemical parameters of experimental rat serum and its neutralization by black seed (Nigella sativa L.) oil. 
Slovak Journal of Animal Science. 2009;42(2):87-94.

21. Sivapiriya $V$, Venkatraman $S$. Effects of dimethoate $(\mathrm{O}, \mathrm{O}$-dimethyl $\mathrm{S}$-methy carbamoyl methyl phosphorodithioate) and ethanol in antioxidant status of liver and kidney of experimental mice. Pesticide Biochemistry and Physiology. 2006;85(2):115-21.

22. El-Damaty $\mathrm{E}$, et al. Biochemical and histopathological effects of systemic pesticides on some functional organs of male albino rats. J Appl Sci Res. 2012;8(11):5459-69.

23. Salim A, et al. Influence of pomegranate (Punica granatum L.) on dimethoate induced hepatotoxicity in rats. Int $\mathrm{J}$ Biol Biomol Agr Food Biotechn Eng. 2014;8(8):908-13.

24. Yeragi S, Rana A, Koli V. Effect of pesticides on protein metabolism of mud skipper Boleophthalmus dussumieri. Journal of Ecotoxicology and Environmental Monitoring. 2003;13(3):211-4.

25. Alarami AM. Histopathological Changes in the Liver and Kidney of Albino Mice on Exposure to Insecticide, Dimethoate. Int J Curr Microbiol App Sci. 2015;4(7):287-300.

26. Ajani E, et al. Toxicological implications of continuous administration of aqueous leaves extract of hydrocotyl bonariensis in rats. Archives of Applied Science Research. 2011;3(5):471-8.

27. Saafi EB, et al. Protective effect of date palm fruit extract (Phoenix dactylifera L.) on dimethoate induced-oxidative stress in rat liver. Experimental and Toxicologic Pathology. 2011;63(5):433-41.

28. Zulkhairi A, et al. Alpha lipoic acid posses dual antioxidant and lipid lowering properties in atherosclerotic-induced New Zealand White rabbit. Biomedicine and Pharmacotherapy. 2008;62(10):716-22.

29. Odabasoglu $F$, et al. $\alpha$-Lipoic acid has anti-inflammatory and anti-oxidative properties: An experimental study in rats with carrageenan-induced acute and cotton pellet-induced chronic inflammations. British Journal of Nutrition. 2011;105(1):31-43.

30. Hamzawy MA, et al. Hepatoprotective effect of estradiol and-Lipoic Acid in Rats. Global Journal of Pharmacology. 2014;8(4):694-702

31. Bae $E H$, et al. Effects of $\alpha$-lipoic acid on ischemia-reperfusion-induced renal dysfunction in rats. American Journal of Physiology-renal Physiology. 2008;294(1):F272-80.

32. Valdecantos MP, et al. Lipoic acid administration prevents nonalcoholic steatosis linked to long-term high-fat feeding by modulating mitochondrial function. The Journal of Nutritional Biochemistry. 2012;23(12):1676-84.

33. Evans JL, Goldfine ID. $\alpha$-Lipoic acid: a multifunctional antioxidant that improves insulin sensitivity in patients with type 2 diabetes. Diabetes Technology and Therapeutics. 2000;2(3):401-13.

34. Hong YS, et al. The inhibitory effects of lipoic compounds on mammalian pyruvate dehydrogenase complex and its catalytic components. Free Radical Biology and Medicine. 1999;26(5-6):685-94.

35. Lone $Y$, et al. Dimethoate Induced Alteration in Serum Biochemical Parameters in Rattus Rattus. World Journal of Pharmaceutical Research. 2017;6523(14):60313.

36. Amara IB, et al. Dimethoate induced oxidative damage and histopathological changes in lung of adult rats: modulatory effects of selenium and/or vitamin $\mathrm{E}$. Biomedical and Environmental Sciences. 2012;25(3):340-51.
37. Surana B, Mehta J, Seshadri S. Toxicological effects of QUinolphos and its subsequent reversal by using root extract of Withania somnifera and leaf pulp of Aloe barbadensis. J Indian Soc Toxicol. 2009;4(2):1-5.

38. Hai DQ, Varga I, Matkovics B. Effects of an organophosphate on the antioxidant systems of fish tissues. Acta Biologica Hungarica. 1995;46(1):39-50.

39. Poovala VS, Huang H, Salahudeen AK. Role of reactive oxygen metabolites in organophosphate-bidrin-induced renal tubular cytotoxicity. Journal of the American Society of Nephrology. 1999;10(8):1746-52.

40. Takaoka M, et al. Protective Effect of $\alpha$-LIPOIC Acid Against Ischaemic Acute Renal Failure In Rats. Clinical and Experimental Pharmacology and Physiology. 2002;29(3):189-94.

41. Şehirli Ö, et al. $\alpha$-Lipoic acid protects against renal ischaemia-reperfusion injury in rats. Clinical and Experimental Pharmacology and Physiology. 2008;35(3):24955.

42. Koga $H$, et al. New $\alpha$-lipoic acid derivative, DHL-HisZn, ameliorates renal ischemia-reperfusion injury in rats. Journal of Surgical Research. 2012;174(2):352-8.

43. El-Saad AA, Elgerbed M. Dimethoate induced hepatotoxicity in rats and the protective roles of vitamin E and N-acetylcysteine. Egypt J Exp Biol. 2010;6(2):21930 .

44. Adham $\mathrm{K}$, et al. Environmental stress in lake maryut and physiological response of Tilapia zilli Gerv. Journal of Environmental Science and Health Part A. 1997:32(9-10):2585-98.

45. Zaahkouk SAM, et al. Carbamate toxicity and protective effect of vit. A and vit. E on some biochemical aspects of male albino rats. Egyptian Journal of Hospital Medicine. ;1:60-77.

46. Lucic A, et al. The effect of dichlorvos treatment on butyrylcholinesterase activity and lipid metabolism in rats. Arhiv za Higijenu Rada I Toksikologiju/ Archives of Industrial Hygiene and Toxicology. 2002;53(4):275-82.

47. Kalender S, et al. Diazinon-induced hepatotoxicity and protective effect of vitamin $\mathrm{E}$ on some biochemical indices and ultrastructural changes. Toxicology. 2005:211(3):197-206.

48. Zhang Y, et al. Amelioration of Lipid Abnormalities by $\alpha$-Lipoic acid Through Antioxidative and Anti-Inflammatory Effects. Obesity. 2011;19(8):1647-53.

49. Budin SB, et al. Alpha lipoic acid prevents pancreatic islet cells damage and dyslipidemia in streptozotocin-induced diabetic rats. The Malaysian Journal of Medical Sciences: MJMS. 2007;14(2):47.

50. Morakinyo AO, Awobajo FO, Adegoke OA. Effects of alpha lipoic acid on blood lipids, renal indices, antioxidant enzymes, insulin and glucose level in streptozotocin-diabetic rats. Biology and Medicine. 2013;5:26.

Cite this article: Abdelsalam HM. Mitigative Effects of Alpha-lipoic Acid on the Toxicity of Dimethoate in Male Rats. Int J Clin Exp Physiol. 2019;6(2):55-60. 